\title{
AIDS-Related Anal Carcinoma
}

National Cancer Institute

\section{Source}

National Cancer Institute. AIDS-Related Anal Carcinoma. NCI Thesaurus. Code C9278.

A carcinoma arising from the anus and occurring in HIV-positive patients. Homosexual HIV-positive men have an increased risk of developing anal squamous cell carcinoma in comparison to the general male population. Sexually transmitted human papillomavirus is detected in the majority of the cases. 